\title{
Underlying principle of efficient propulsion in flexible plunging foils
}

\author{
Xiao-Jue Zhu $\cdot$ Guo-Wei He $\cdot$ Xing Zhang
}

Received: 4 September 2014 / Revised: 15 September 2014 / Accepted: 30 December 2014

(C)The Chinese Society of Theoretical and Applied Mechanics and Springer-Verlag Berlin Heidelberg 2014

\begin{abstract}
Passive flexibility was found to enhance propulsive efficiency in swimming animals. In this study, we numerically investigate the roles of structural resonance and hydrodynamic wake resonance in optimizing efficiency of a flexible plunging foil. The results indicates that (1) optimal efficiency is not necessarily achieved when the driving frequency matches the structural eigenfrequency; (2) optimal efficiency always occurs when the driving frequency matches the wake resonant frequency of the time averaged velocity profile. Thus, the underlying principle of efficient propulsion in flexible plunging foil is the hydrodynamic wake resonance, rather than the structural resonance. In addition, we also found that whether the efficiency can be optimized at the structural resonant point depends on the strength of the leading edge vortex relative to that of the trailing edge vortex. The result of this work provides new insights into the role of passive flexibility in flapping-based propulsion.
\end{abstract}

Keywords Flexibility · Propulsive efficiency · Structural resonance $\cdot$ Hydrodynamic wake resonance

\section{Introduction}

Wings or fins are the most commonplace structures used by animals for thrust production. Large passive or active deformation is often borne by these propulsive structures when they flap in air or water [1-4]. Recently, it has been reported that passive flexibility of wing/fin can result in the enhancement of thrust production or propulsive efficiency [5].

The project was supported by the National Natural Science Foundation of China (11232011, 11021262, and 11023001) and the National Basic Research Program of China (2013CB834100).

X.-J. Zhu $\cdot$ G.-W. He $\cdot$ X. Zhang (四)

LNM, Institute of Mechanics,

Chinese Academy of Sciences, 100190 Beijing, China

e-mail: zhangx@1nm.imech.ac.cn
In some works, the optimization of propulsive performance due to flexibility was rationalized by the occurrence of structural harmonic or super-harmonic resonance [6]. In this regard, however, conflicting results have been obtained. Other researchers have found that the optimal performance was achieved at a much lower flapping frequency than the first structural eigenfrequency and the beneficial effect of flexibility was explained by the formation of a streamlined foil shape due to deformation [7].

In the study of flapping wings/fins, another interesting topic in parallel with the aforesaid one is the connection between propulsive performance and wake structure. By using a linear stability analysis of the time averaged wakes behind a flapping-foil, Triantafyllou [8] proposed that the peak efficiency was achieved when the flapping frequency coincided with the hydrodynamic wake resonant frequency (i.e., the frequency for maximum spatial growth of instabilities in the averaged velocity profile). Under this principle, the Strouhal number for efficient propulsion was found to lie in the narrow range of $0.2-0.4$. Recently, the validity of this principle has also been demonstrated in flapping-foils with prescribed active deformations [9] and pitching foils with passive flexibility [10]. In this work, we conduct numerical simulation to compute the propulsive efficiency in a flexible foil which is driven at its leading edge by a plunging motion and is placed in a uniform oncoming flow. Linear analysis is conducted to find the wake resonance frequency. We will show that for a flexible plunging foil, the wake hydrodynamic resonance is the underlying principle for efficient propulsion, whereas the structural resonance is not the necessary condition for optimizing the efficiency.

The rest of the paper is arranged as follows. In Sect. 2, we introduce the model problem and numerical methods. In Sect. 3.1, the relation between the structural resonance and the propulsive efficiency is explored. In Sect.3.2, we study the relation between the wake hydrodynamic resonance and the propulsive efficiency. Finally, some conclusions are drawn in Sect. 4. 


\section{Model problem and numerical methods}

We consider the interaction between an inextensible flexible filament and a two-dimensional, incompressible and laminar flow. The leading-edge of the filament is driven by a harmonic plunging motion and is also clamped (see Fig. 1). The filament is placed in the free stream with a uniform velocity.

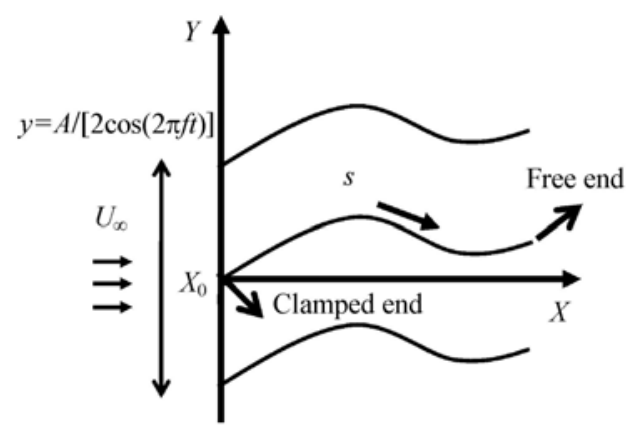

Fig. 1 Schematic depiction of the model problem

\subsection{Governing equations}

The motions of the fluid and the filament are governed by the Navier-Stokes equations coupled with a geometrically nonlinear structural equation [11-13]

$\frac{\partial \boldsymbol{u}}{\partial t}+\nabla \cdot(\boldsymbol{u u})=-\nabla p+\frac{1}{\operatorname{Re}} \nabla^{2} \boldsymbol{u}+\boldsymbol{f}$,

$\nabla \cdot \boldsymbol{u}=0$

$\beta \frac{\partial^{2} \boldsymbol{X}}{\partial t^{2}}-\frac{\partial}{\partial s}\left(\zeta \frac{\partial \boldsymbol{X}}{\partial s}\right)+\frac{\partial^{2}}{\partial s^{2}}\left(\gamma \frac{\partial^{2} \boldsymbol{X}}{\partial s^{2}}\right)=-\boldsymbol{F}$,

$\frac{\partial \boldsymbol{X}}{\partial s} \cdot \frac{\partial \boldsymbol{X}}{\partial s}=1$

where $\boldsymbol{u}, p$, and $\boldsymbol{X}=\left(X^{(1)}, X^{(2)}\right)$ are the fluid velocity, fluid pressure and displacement of the filament respectively; $f$ and $\boldsymbol{F}$ are the Eulerian and Lagrangian force densities which represent the interactions between the fluid and the structure.

Equations (1)-(4) are written in a dimensionless form, which is obtained by scaling space and time with the filament length $L$ and $L / U_{\infty}$ respectively, where $U_{\infty}$ is the oncoming flow velocity. The dimensionless numbers in Eqs. (1)-(4) are: $R e=\rho_{\mathrm{f}} U_{\infty} L / \mu, \gamma=B /\left(\rho_{\mathrm{f}} U_{\infty}^{2} L^{3}\right), \zeta=T /\left(\rho_{\mathrm{f}} U_{\infty}^{2} L\right)$, $\beta=\rho_{\mathrm{s}} /\left(\rho_{\mathrm{f}} L\right)$, which are the Reynolds number, dimensionless bending rigidity, dimensionless tension and mass ratio, respectively. Here $\rho_{\mathrm{f}}$ and $\rho_{\mathrm{s}}$ are the densities of the fluid and the filament respectively; $\mu$ is the dynamic viscosity of the fluid; $B$ and $T$ are the dimensional bending rigidity and tension of the filament.

By following the same scaling procedure, the dimensionless form of the prescribed position of the leading-edge becomes $\bar{y}(t)=\frac{1}{2} \bar{A} \cos (2 k t)$. Here $\bar{A}=A / L$ is the dimensionless plunging amplitude (with $A$ being the dimensional plunging amplitude). $\quad k=(\pi f L) / U_{\infty}$ is the reduced frequency.

\subsection{Numerical method for FSI simulation}

The simulations are performed by using the immersed boundary method for the fluid flow and the finite difference method for the motion of the structure. The inextensibility condition of the filament is enforced by solving a Poisson equation for $\zeta$ [11-13]. Extensive validations of the immersed boundary solver can be found in Ref. [14] (on flows past rigid objects) and [15] (on FSI problems). For the simulations of this work, a rectangular computational domain of size $20 L \times 12 L$ used, with the grid width being $0.02 L$. The time steps are chosen such that the maximum $C F L$ number never exceeds 0.5. Systematic studies have been conducted to ensure mesh convergence of the solutions obtained.

The boundary and initial conditions for the FSI simulation are as follows. At $s=0$ (the leading-edge of the structure), a boundary condition which blends the vertically forced oscillation and the clamped condition is enforced, i.e.,

$\boldsymbol{X}=(0, \bar{y}(t))^{\mathrm{T}}$,

$\frac{\partial \boldsymbol{X}}{\partial s}=(1,0)^{\mathrm{T}}$.

At $s=1$ (the trailing-edge of the structure), a free-end condition is imposed, i.e.,

$\frac{\partial^{2} \boldsymbol{X}}{\partial s^{2}}=(0,0)^{\mathrm{T}}$,

$\frac{\partial^{3} \boldsymbol{X}}{\partial s^{3}}=(0,0)^{\mathrm{T}}$.

For the fluid, the zero-velocity condition is enforced at the four outer boundaries. The motions of the fluid and the structure are coupled at their interface by the no-slip condition and the relation between the Lagrangian and Eulerian force densities, i.e.,

$\int_{V} \boldsymbol{u}(\boldsymbol{x}, t) \delta(\boldsymbol{x}-\boldsymbol{X}) \mathrm{d} \boldsymbol{x}=\mathrm{d} \boldsymbol{X} / \mathrm{d} t$,

$\boldsymbol{f}(\boldsymbol{x}, t)=\int_{s} \boldsymbol{F}(\boldsymbol{X}(s), t) \delta(\boldsymbol{x}-\boldsymbol{X}(s)) \mathrm{d} s$,

where $\delta$ is the discrete delta function. The initial condition for the structure is

$\boldsymbol{X}(s, 0)=(s, \bar{A})^{\mathrm{T}}$,

$\mathrm{d} \boldsymbol{X} / \mathrm{d} t(s, 0)=(0,0)^{\mathrm{T}}$.

A uniform velocity everywhere is the initial condition for the fluid.

2.3 Numerical method for linear stability analysis

In the linear spatial stability analysis, first an onedimensional (locally parallel) base flow, $\boldsymbol{U}=[U(y), 0]$, is identified [8]. Then a small perturbation is superimposed onto the base flow and the resultant velocity is substituted into the Navier-Stokes equations. After linearization and assuming a travelling wave form for the perturbation, the OrrSommerfeld (O-S) equation for the complex amplitude of the 
velocity perturbation (or equivalently, stream function perturbation) can be derived. The O-S equation for the complex amplitude, $\varphi(y)$, of the stream function perturbation, $\psi(x, y, t)$, can be written as (with $D=\mathrm{d} / \mathrm{d} y$ )

$\left\{\left(D^{2}-\alpha^{2}\right)^{2}-\mathrm{i} \operatorname{Re}\left[(\alpha U-\omega)\left(D^{2}-\alpha^{2}\right)-\alpha U^{\prime \prime}\right]\right\} \varphi=0$,

where

$\psi(x, y, t)=\varphi(y) \mathrm{e}^{\mathrm{i}(\alpha x-\omega t)}$,

$u(x, y, t)=\frac{\partial \psi}{\partial y}$,

$v(x, y, t)=-\frac{\partial \psi}{\partial x}$

Here $\boldsymbol{u}=(u, v)$ is the velocity perturbation. $\alpha=\omega / c$ is the complex wavenumber, where $c$ is the complex phase velocity. For the spatial stability analysis, the frequency $\omega$ is real [16]. If the imaginary part of the complex wavenumber becomes negative, the disturbance will grow in space (downstream of the perturbations). The spatial stability problem forms a nonlinear eigenvalue problem (with the eigenvalues being $\alpha$ and the associated eigenmodes being $\varphi$ ).

To solve the nonlinear eigenvalue problem, the O-S equation is expanded in powers of $\alpha$ and then discretized. The discretized nonlinear eigenvalue problem can be casted into the companion matrix form $[17,18]$ to form a linear eigenvalue problem which can be solved using standard techniques. The resulting eigenvalue problem for $\alpha$ is then solved by choosing $\omega$ to be real numbers. Two hundred evenly spaced points were used to represent the velocity profile $U(y)$. Horizontal velocities at these points were computed by interpolations. No significant difference in the solution of the Orr-Sommerfeld equation was found by using 400 points.

We validate our O-S solver with a benchmark Poiseuille flow calculated by Bridges and Morris [18]. By choosing $\omega=0.26$ and $\operatorname{Re}=6000$, we plot the eigenvalues of $\alpha$ in Fig. 2, which agree very well with the results in Ref. [18]. The most unstable seven modes are listed and compared in Table 1.

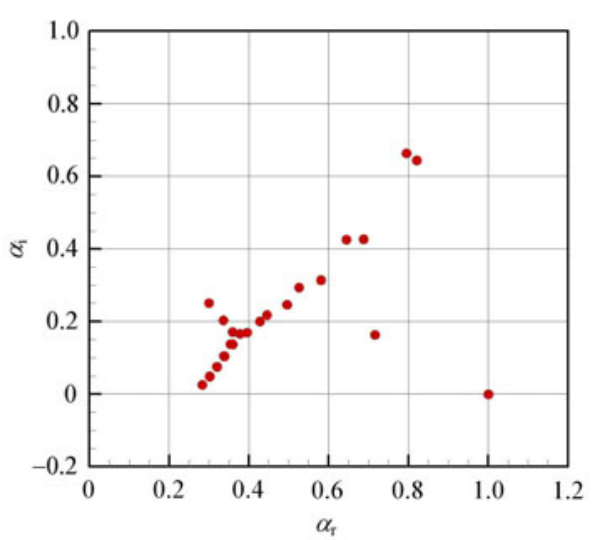

Fig. 2 Eigenvalues on complex $\alpha$ plane for a Poiseuille flow
Table 1 Seven most unstable modes compared with Bridges and Morris [18]

\begin{tabular}{lll}
\hline Mode & Bridges and Morris [18] & Present \\
\hline 1 & $1.00470+0.00086 \mathrm{i}$ & $1.00470+0.00086 \mathrm{i}$ \\
2 & $0.28323+0.02538 \mathrm{i}$ & $0.28323+0.02538 \mathrm{i}$ \\
3 & $0.30165+0.04886 \mathrm{i}$ & $0.30165+0.04886 \mathrm{i}$ \\
4 & $0.31975+0.07532 \mathrm{i}$ & $0.31975+0.07532 \mathrm{i}$ \\
5 & $0.33745+0.10492 \mathrm{i}$ & $0.33745+0.10492 \mathrm{i}$ \\
6 & $0.35456+0.13782 \mathrm{i}$ & $0.35456+0.13782 \mathrm{i}$ \\
7 & $0.37090+0.17425 \mathrm{i}$ & $0.37090+0.17425 \mathrm{i}$ \\
\hline
\end{tabular}

\section{Result and discussions}

There are five control parameters in this problem: $\operatorname{Re}, \bar{A}, k$, $\beta$, and $\gamma$. In this work, simulations are conducted by fixing the Reynolds number to 200 while choosing different combinations of relative amplitude and mass ratio.

Some important physical quantities used to characterize the propulsive performance and vortex shedding of the plunging filament are defined as follows. The effective amplitude $A_{\text {eff }}$ is defined as the peak-to-peak amplitude measured at the trailing-edge and the amplitude ratio is defined as $A_{\text {eff }} / A$. The (dimensionless) averaged thrust $\bar{F}_{T}$ is defined as the average thrust evaluated when periodicity is reached

$\bar{F}_{T}=\frac{1}{T_{0}} \int_{0}^{T_{0}} F^{(1)} \mathrm{d} t$,

where $T_{0}=\pi / k$ is the (dimensionless) period of the plunging motion. The Strouhal number based on the leading-edge amplitude is defined as

$S t_{A}=(f A) / U_{\infty}=(k \bar{A}) / \pi$.

Similarly, the Strouhal number based on trailing-edge amplitude is defined as

$S t_{A}^{*}=\left(f A_{\mathrm{eff}}\right) / U_{\infty}=(k \bar{A} / \pi)\left(A_{\mathrm{eff}} / A\right)$.

The time-averaged power input $\bar{P}_{\text {in }}$ (which is needed for actuating the plunging motion), can be computed by

$\bar{P}_{\text {in }}=\frac{1}{T_{0}} \int_{0}^{T_{0}} F^{(2)} \dot{y}(t) \mathrm{d} t$,

where $F^{(2)}$ is the total vertical force exerted on the filament. Lastly, the propulsive efficiency in the filament is defined as

$\eta=\bar{F}_{T} U_{\infty} / \bar{P}_{\text {in }}$.

The values of the some parameters used in the simulation are listed in Table 2. In this work, the Strouhal number is set to 0.2 , which lies in the region of optimized efficiency for rigid flapping foils. It should be noted that the current stability analysis can only be applied to one-dimensional base flow. This selection of Strouhal number also avoids the occurrence of oblique wakes (which are intrinsically twodimensional) [13]. 
Table 2 Values of some parameters used in the simulations

\begin{tabular}{lllllll}
\hline Series & $R e$ & $\bar{A}$ & $\beta$ & $\gamma$ & $k$ & $S t_{A}$ \\
\hline A & 200 & 0.2 & 2.0 & $10^{-1}-10^{4}$ & $\pi$ & 0.2 \\
B & 200 & 0.4 & 0.2 & $10^{-2}-10^{4}$ & $\pi / 2$ & 0.2 \\
\hline
\end{tabular}

\subsection{Role of structural resonance in optimizing efficiency}

In this section, we will explore the role of structural resonance (between the forcing and the natural frequency) in optimizing efficiency.

First, we define the first frequency ratio, $\bar{\omega}=\omega_{\mathrm{f}} / \omega_{1}=$ $2 \pi f / \omega_{1}$, where $\omega_{\mathrm{f}}$ is the driving angular frequency at the leading edge and $\omega_{1}$ is the first natural angular frequency of the system. For the cases of large mass ratios (where the influence of outside fluid can be neglected), the natural frequencies of the system are approximated by those of a clamped-free elastic sheet in vacuum [12]. For such cases (e.g. those in series A), $\omega_{1}$ is calculated analytically by

$\omega_{1}=k_{1}^{2} \frac{U_{\infty}}{L}\left(\frac{\gamma}{\beta}\right)^{1 / 2}$

with

$1+\cosh k_{1} \cos k_{1}=0$

Thus, the reduced frequency becomes

$\bar{\omega}=\frac{2 \pi}{k_{1}^{2}} \frac{f L}{U_{\infty}}\left(\frac{\beta}{\gamma}\right)^{1 / 2}=\frac{1}{k_{1}^{2}} \frac{1}{\bar{A}}\left(\frac{\beta}{\gamma}\right)^{1 / 2}$.

However, for the cases of small mass ratios (such as those in series B), the natural frequencies of the system can be significantly modified due to the presence of outside flow. In this work, we use the first natural frequency of a passive elastic sheet in axial flow as a better approximation to that of the current system. An inviscid "vortex sheet" representation of the wake is then used to compute the natural frequency $\omega_{1}$ of the system [12].

The evolution of the normalized efficiency with increasing reduced forcing frequency is shown in Fig. 3. It is found that the optimal efficiency is not necessarily achieved at the place where the driving frequency matches the first natural frequency. More specifically, in series A (where the mass ratio is larger and the flapping amplitude is smaller), the optimal efficiency occurs at the resonant point; whereas in series $\mathrm{B}$ (where the mass ratio is smaller and the flapping amplitude is larger), the optimal efficiency occurs at the first frequency ratio 0.54 , which is much lower than the resonant point.

It is well known that the propulsive performance of a flapping foil is closely associated with the strength of the vortices shed into the wake. To rationalize the phenomenon above, we investigate the variation of vortex strength with increasing reduced forcing frequency. The vortex strength is quantified by using the circulation [13]. First, the center of vortex is defined as the location of maximum vorticity magnitude. The circulation is then calculated by using the vorticity area integration in a rectangular area surrounding the vortex center. The size of the integration is determined by using Gaussian fits $\mathrm{e}^{\left(-x_{i}^{2} / \sigma_{i}^{2}\right)}$ along the vertical and horizontal axes centered at the positions of the maxima vorticity magnitude. The size of the vortex along the $x$ - and $y$-direction are then defined as $2 \sigma_{i}$.

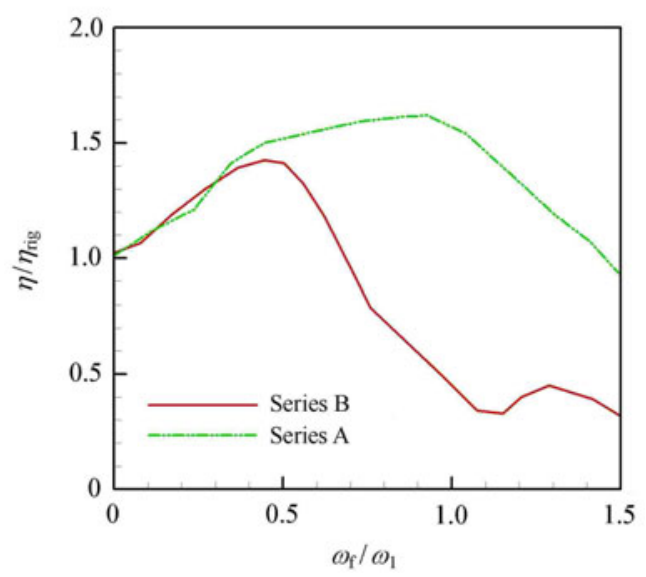

Fig. 3 The normalized efficiency as a function of the first frequency ratio. The efficiency is normalized by the value obtained in the corresponding rigid foil

The vortex strength as a function of the first frequency ratio is plotted in Figs. 4 and 5, for series A and B, respectively. From the simulations we know that the two major contributions of the vorticity shed into the wake are the leading edge vortex and the trailing edge vortex. Later, we will show that whether the optimal efficiency occurs at the resonant point depends on the strength of the trailing edge vortex relative to that of the leading edge vortex. Since the trailing edge amplitude is a good indicator of strength of trailing edge vortex, for reference purpose, the variation of the trailing edge amplitude with increasing reduced forcing frequency is also plotted in these two figures.

From Fig. 4, it is found that in series A, both the vortex intensity and the trailing edge amplitude reach their peaks at the structural resonance point. For this series of cases, the dominating effect of increasing flexibility is the enhancement of trailing edge flapping amplitude. The increase in trailing edge flapping amplitude results in the increase of vorticity contribution from the trailing edge vortex to the wake vortex intensity. (For series A, the comparison of two wake vortex structures, one produced by a very rigid filament and the other produced by a filament at the optimized flexibility, is shown in Fig. 6.) Since the contribution from the trailing edge vortex dominates the wake vortex intensity, the wake vortex intensity is maximized at the resonant point, where the trailing edge amplitude is maximized. Higher vortex intensity in the wake can induce higher jet velocity, which, in turn, increases the thrust and the efficiency. 


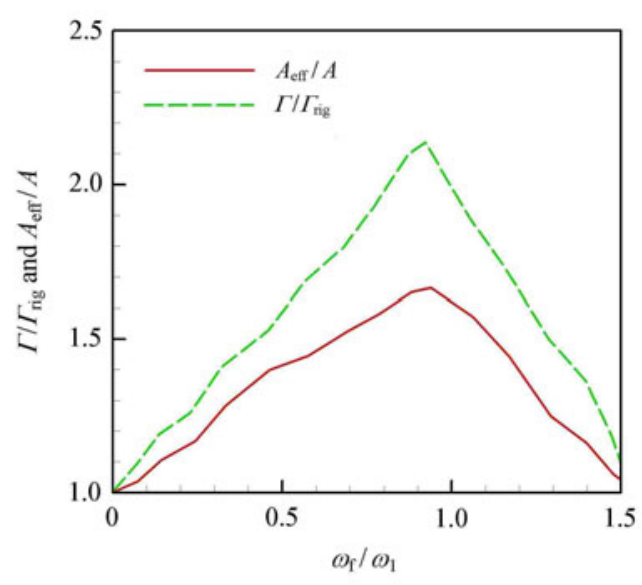

Fig. 4 The normalized trailing edge amplitude and vortex intensity as a function of the first frequency ratio for series A. All quantities are normalized by the values obtained in the corresponding rigid foil

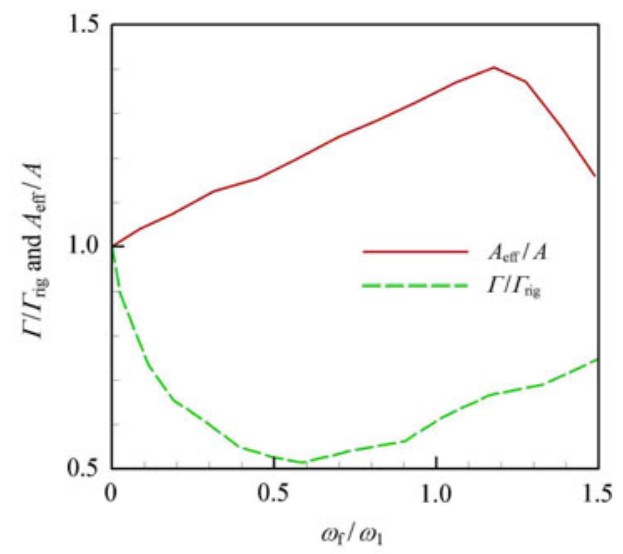

Fig. 5 Wake vortex structures for two cases in series A. a $\gamma=1000$ (very rigid filament); $\mathbf{b}$ The flexibility which optimizes the efficiency

a

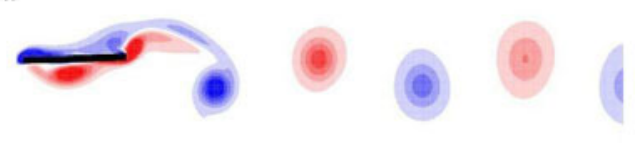

b

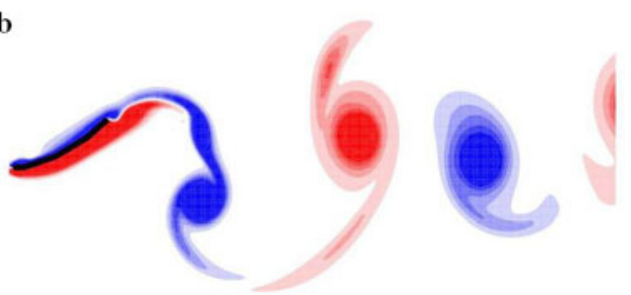

Fig. 6 The normalized trailing edge amplitude and vortex intensity as a function of the first frequency ratio for series B. All quantities are normalized by the values obtained in the corresponding rigid foil
From Fig. 6, it is found that in series B, the trailing edge amplitude still reaches its peak near the structural resonance point, however, no such behavior is observed in the wake vortex intensity. For this series of cases, the dominating effect of increasing flexibility is the weakening of leading edge separation. This can be explained by the formation of a streamlined foil shape as a result of deformation [13]. The weakening of leading edge separation results in the reduction of vorticity contribution from the leading edge vortex to the wake vortex intensity. (For series B, the comparison of two wake vortex structures, one produced by a very rigid filament and the other produced by a filament at the optimized flexibility, is shown in Fig. 7.) Due to the fact that the trailing edge flapping velocity in Fig. 7b increases slightly if compared with that in Fig. 7a, it can be inferred that the decrease of wake vortex intensity in Fig. $7 \mathrm{~b}$ can not be linked with the vorticity production near the trailing edge and must be associated with the decrease of vorticity production at the leading edge. From this figure, we also found that at the reduced frequency of 0.54 , where the optimized efficiency is achieved, the vortex intensity is minimized. The connection between the minimization of vortex intensity and the optimization of efficiency can be rationalized by the fact that the weakening of leading edge separation results in the reduction in input power. Although the decrease of wake vortex intensity may also reduce the thrust production (the thrust for the case in Fig. $7 b$ is only $80 \%$ of that in Fig. 7a), since in series B the former effect is dominating, the efficiency is still enhanced.

a

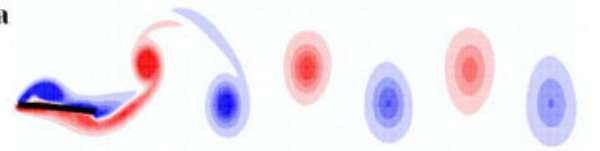

b

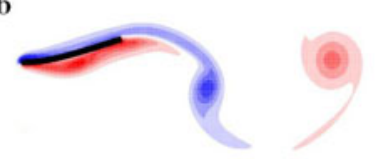

Fig. 7 Wake vortex structures for two cases in series B. a $\gamma=500$ (very rigid filament); b The flexibility which optimizes the efficiency

3.2 Role of wake resonance in optimizing efficiency

We define the wake resonance frequency as $\omega_{\text {wake, }}$, which is determined from the most unstable spatial mode for the average horizontal velocity profiles. These velocity profiles are calculated at one chord length downstream of the trailing edge, where the vertical velocity components are negligibly small. First, the spatial growth rate of the most unstable mode (the most negative imaginary part of the wave number) is computed as a function of real frequency. The frequency corresponding to the maximum spatial amplification is then determined. Figure 8 shows the relationship between the sec- 


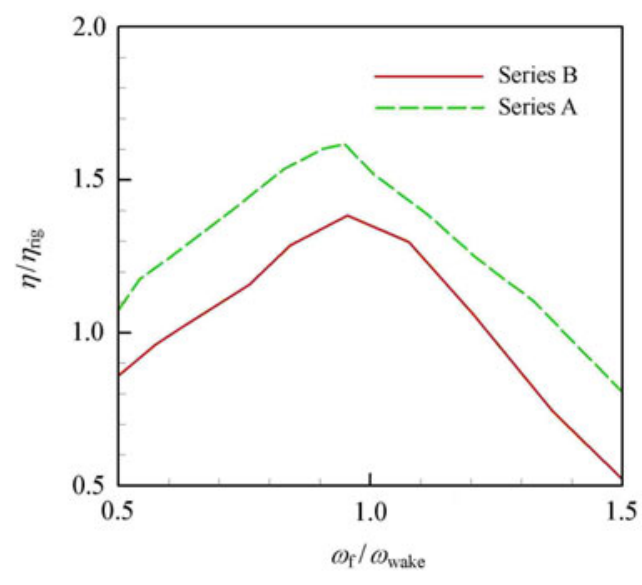

Fig. 8 The normalized efficiency as a function of the first frequency ratio and wake resonance frequency. The efficiency is normalized by the value obtained in the corresponding rigid foil

ond frequency ratio, $\omega_{\mathrm{f}} / \omega_{\text {wake }}$, and the normalized efficiency, for each case in series A and B.

From Fig. 8, it is found that for both series A and series $\mathrm{B}$, a local peak in efficiency occurs when the driving frequency matches the wake resonance frequency. Thus we believe that the underlying principle for efficient propulsion in flexible plunging foil is the hydrodynamic wake resonance, rather than the structural resonance. This finding is consistent with that in the experimental study on flexible pitching foils [10]. The present study provides a further support that the wake resonance principle is valid for both rigid and flexible propulsors.

It was also reported in Ref. [10] that there exists an optimal flexibility which globally maximizes the efficiency. This optimal flexibility can be reached by tuning the structural resonant frequency to the wake resonant frequency. In other words, the globally optimized efficiency can be achieved when $\omega_{\mathrm{f}}=\omega_{\text {wake }}=\omega_{1}$. In the present study, we confirm that the maximal efficiency for series A (where the aforesaid condition is met) is higher than that for series B (where the aforesaid condition is not met). However, further investigation is still needed to verify whether the optimal flexibility for series A is indeed the flexibility which globally maximizes the efficiency.

\section{Conclusions}

To investigate the influence of structural resonance and hydrodynamic wake resonance on the efficiency of a flexible propulsor, we consider a simplified problem in which the two-dimensional laminar flow interacts with a plunging filament. The control parameters in the model problem are: the flapping Reynolds number $R e$, the dimensionless oscillating amplitude $\bar{A}$, the mass ratio $\beta$, the dimensionless driving frequency $k$ and the dimensionless bending rigidity $\gamma$. In this study, we fix the Reynolds number to 200 and consider two different combinations of dimensionless oscillating amplitude, mass ratio, and dimensionless driving frequency. For each combination aforesaid, we perform a series of simulations by varying the dimensionless bending rigidity within a certain range while keeping other parameters unchanged.

It is shown that for the two series of cases studied, the optimal efficiency is not necessarily achieved when the driving frequency matches the structural resonance frequency. When there is little leading edge separation, the optimized efficiency occurs at the structural resonant point. However, when there exists substantial leading edge separation, the optimized efficiency can occur when the driving frequency is much lower than the structural resonance frequency. On the other hand, for both series of cases, it is found that the optimal efficiency is always achieved when the driving frequency is close to the wake resonance frequency. This result provides the further support that the hydrodynamic wake resonance principle is also valid in flexible propulsors.

\section{References}

1 Liu, G., Ren, Y., Zhu, J.Z., et al.: Thrust producing mechanisms in ray-inspired underwater vehicle propulsion. Theor. Appl. Mech. Lett. Doi: 10.1016/j.taml.2014.12.004 (2015)

2 Tang, C., Lu, X.Y.: Propulsive performance of two- and threedimensional flapping flexible plates. Theor. Appl. Mech. Lett. Doi: 10.1016/j.taml.2015.01.010 (2015)

3 Wang, S.Z., Zhang, X., He, G.W., et al.: Numerical simulation of unsteady flows over a slow-flying bat. Theor. Appl. Mech. Lett. Doi: 10.1016/j.taml.2015.01.006 (2015)

4 Yu, Y.L., Guan, Z.W.: Learning from bat: aerodynamics of actively morphing wing. Theor. Appl. Mech. Lett. Doi: 10.1016/j.taml.2015.01.009 (2015)

5 Shyy, W., Anon, H., Chimakurthi, S.K., et al.: Recent progress in flapping wing aerodynamics and aeroelasticity. Prog. Aerosp. Sci. 46, 284-327 (2010)

6 Michelin, S.B., Llewellyn Smith, S.G.: Resonance and propulsion performance of a heaving flexible wing. Phys. Fluids 21, 071902 (2009)

7 Ramananarivo, S., Godoy-Diana, R., Thiria, B.: Rather than resonance, flapping wing flyers may play on aerodynamics to improve performance. Proc. Natl. Acad. Sci. U.S.A. 108, 5964-5969 (2011).

8 Triantafyllou, G.S., Triantafyllou, M.S., Grosenbaugh, M.A.: Optimal thrust development in oscillating foils with application to fish propulsion. J. Fluids Struct. 7, 205-224 (1993)

9 Moored, K.W., Dewey, P.A., Smits A.J., et al.: Hydrodynamic wake resonance as an underlying principle of efficient unsteady propulsion. J. Fluid Mech. 708, 329-348 (2012)

10 Moored, K.W., Dewey, P.A., Boschitsch, B.M., et al.:Linear instability mechanisms leading to optimally efficient locomotion with flexible propulsors. Phys. Fluids 26, 041905 (2014)

11 Huang, W.X., Shin, S.J., Sung, H.J.: Simulation of flexible filaments in a uniform flow by the immersed boundary method. J. Comput. Phys. 226, 2206-2228 (2007)

12 Zhu, X.J., He, G.W., Zhang, X.: Numerical study on hydrodynamic effect of flexibility in a self-propelled plunging foil. Compt. Fluids 97, 1-20 (2014)

13 Zhu, X.J., He, G.W., Zhang, X.: How flexibility affects the wake symmetry properties of a self-propelled plunging foil. J. 
Fluid Mech. 571, 164-183 (2014)

14 Wang, S., Zhang, X.: An immersed boundary method based on discrete stream function formulation for two- and threedimensional incompressible flows. J. Comput. Phys. 230, 3479-3499 (2011)

15 Zhu, X.J., He, G.W., Zhang, X.: An improved direct-forcing immersed boundary method for fluid-structure interaction simulations. J. Fluids Eng. 136, 040903 (2014)
16 Mattingly, G.E., Criminale, W.O.: The stability of an incompressible two-dimensional wake. J. Fluid Mech. 51, 233-272 (1972)

17 Orszag, S.A.: Accurate solution of the Orr-Sommerfeld stability equation. J. Fluid Mech. 50, 689-703 (1971)

18 Bridges, T.J., Morris, P.J.: Differential eigenvalue problems in which the parameter appears nonlinearly. J. Comput. Phys. 55, 437-460 (1984) 